\title{
Achieving robust global bandwidth along with bypassing geo-restriction for internet users
}

\author{
Gazi Zahirul Islam, Aman Ullah Juman, Al Nahian Bin Emran, Md. Abbas Ali Khan, \\ Md. Fokhray Hossain, Md. Tarek Habib \\ Department of Computer Science and Engineering, Daffodil International University, Bangladesh
}

\begin{tabular}{|c|c|}
\hline Article Info & ABSTRACT \\
\hline Article history: & \multirow{12}{*}{$\begin{array}{l}\text { Not all Internet Service Providers provide a sufficient amount of bandwidth } \\
\text { to their users. Although the amount of local bandwidth is reasonable, global } \\
\text { bandwidth is not satisfactory at all. Based on bandwidth allocation, location } \\
\text { and price; service providers capped their users' global bandwidth i.e., } \\
\text { reducing global internet speed. As a consequence, we observe severe global } \\
\text { bandwidth limitation among Internet users. In this article, we implement a } \\
\text { flexible and pragmatic solution for Internet users to bypass global bandwidth } \\
\text { restriction. To achieve robust global bandwidth, we utilize a combination of } \\
\text { communication technologies and devices namely, Internet Exchange Point, } \\
\text { Virtual Private Network, chain VPN technology etc. In this project, we show } \\
\text { that internet speed of international route i.e., global bandwidth can enhance } \\
\text { significantly if there are multiple ISPs use a common IXP and at least one of } \\
\text { those ISPs provides pleasant global bandwidth. Usually, regional ISPs use a } \\
\text { common IXP to route their local traffic using local bandwidth within the } \\
\text { region without wasting global bandwidth. We show that using our proposed } \\
\text { method global internet speed of a user can raise several times effectively } \\
\text { utilizing assigned local bandwidth. In addition, we also implement a geo- } \\
\text { restriction bypassing technique integrating an offshore ISP with local ISP } \\
\text { using VPN. Thus, we enjoy tremendous Internet speed along with } \\
\text { unrestricted access to the websites. }\end{array}$} \\
\hline Received Aug 11, 2019 & \\
\hline Revised Sep 21, 2019 & \\
\hline Accepted Oct 19, 2019 & \\
\hline Keywords: & \\
\hline BDIX & \\
\hline Firewall & \\
\hline Global bandwidth & \\
\hline ISP & \\
\hline IXP & \\
\hline Local bandwidth & \\
\hline VPN & \\
\hline
\end{tabular}

Copyright $@ 2020$ Institute of Advanced Engineering and Science. All rights reserved.

\section{Corresponding Author:}

Gazi Zahirul Islam,

Department of Computer Science and Engineering,

Daffodil International University, Dhaka-1207, Bangladesh.

Email: zahir.cse@daffodilvarsity.edu.bd, zahircuet@gmail.com

\section{INTRODUCTION}

IT is undeniable that the Internet is a ground-breaking technology in this century. We hardly think of a day without using the internet. There's hardly any sphere in human life left where the internet hasn't brought any change yet. From the outer space to the deepest trench of the world, the internet has brought the world to our finger's end. For the greater welfare of mankind, the Internet should reach every people's hand at their greatest conveniences. But there are too many deficiencies in our system that has been a hindrance to spreading the internet at a convenient cost. Pointing out all of the deficiencies of the internet is not the main focus of this paper. In this paper, we have come up with a solution to make the Internet handy. In many developing countries like Bangladesh, Internet bandwidth is not well distributed with a well-maintained pricing list. Pricing of packages varies from one ISP to another which is making conundrum to the users. Peoples are not getting proper bandwidth by paying money which they yearn. Somewhere they are getting fair service for internet packages by paying a decent amount of money and somewhere they cannot think of getting the same service with the same amount of money. So, focusing on the pricing variations, here we find a convenient solution to get superior global speed by using the Internet Exchange Point (IXP). 
A direct connection between two devices is naturally the most efficient way to exchange data. If two networks in the same area need to exchange data but they are not directly connected or not connected to a local exchange point, they will pass their data through their upstream Internet providers [15]. This incurs added costs and delays, as upstream providers charge by bandwidth capacity or utilization, and the traffic often pass through other cities before reaching its destination. Internet exchange point is invented to affect the speed of the internet and give a whole new infrastructure of communication.

To implement this project, we have studied several promising articles such as $[1,6,7,27,28]$ based on Internet Exchange Point. Here in Bangladesh year by year internet users are increasing [30] and several IoT base systems are developing [31]. These articles focus on the opportunities and challenges of setting up the IXPs in the developing countries in Asia and Africa. The details outcome of the above papers and other notable resources are explained in section 4. We thoroughly investigate seven ISPs i.e., [20-26] to complete the project successfully. Out of the seven ISPs, six are located in the capital city Dhaka and one ISP named 'Digital Ocean' [26] which employed for chain VPN technology located in the New York City of the USA. We have also gathered important statistics from several official websites $[3,4,8,18,19]$ regarding the telecommunication growth, prospects and future challenges in Bangladesh.

The rest of the paper is organized as follows. We first describe the role and structure of IXP in section 2 and then discuss the background of the project in section 3. We elaborate related works and motivation in section 4. Section 5 implements the core objective of this project i.e., enhancement of users' global bandwidth. Section 4 contains performance evaluation and cost-benefit analysis. In section 7 we add a firewall bypassing technique with our proposed bandwidth enhancement mechanism. This firewall bypassing technique satisfy our second objective of this project i.e., unrestricted access to the Internet. Constraints and recommendations are discussed in section 8. Finally, section 9 concludes the paper.

\section{ROLE AND STRUCTURE OF IXP}

An Internet exchange point (IXP) is a physical location through which Internet infrastructure companies such as Internet Service Providers (ISPs) and CDNs (Content Distribution Networks) connect with each other [16]. These locations exist on the "edge" of different networks and allow network providers to share transit outside their own network. Without IXP we face the following problems:

a) The data have to travel a long distance

b) The ISPs have to pay a huge amount of money to the upstream provider

c) The internal traffic has to go outside the country thereby increases the risk of security

d) Higher latency

e) Complex route peering policy

Normally, an ISP peers with another ISP which establish the upstream route. Some peering route may be too long to travel. However, ISP could reduce that long distance by agreeing with the shortest possible peered ISP. IXP can provide shortest route information to its connected ISPs and thereby reduces the latency.

A typical IXP schematic diagram is shown in Figure 1. Here IXP servers as a common exchange point of the internet where Internet Service Providers (ISPs) are interconnected via IXP. ISPs are also connected to each other through the global internet cloud [1]. An IXP interconnects ISPs in a region or country, allowing them to exchange domestic Internet traffic locally without having to send those data across multiple international gateways to reach their final destination $[13,14]$.

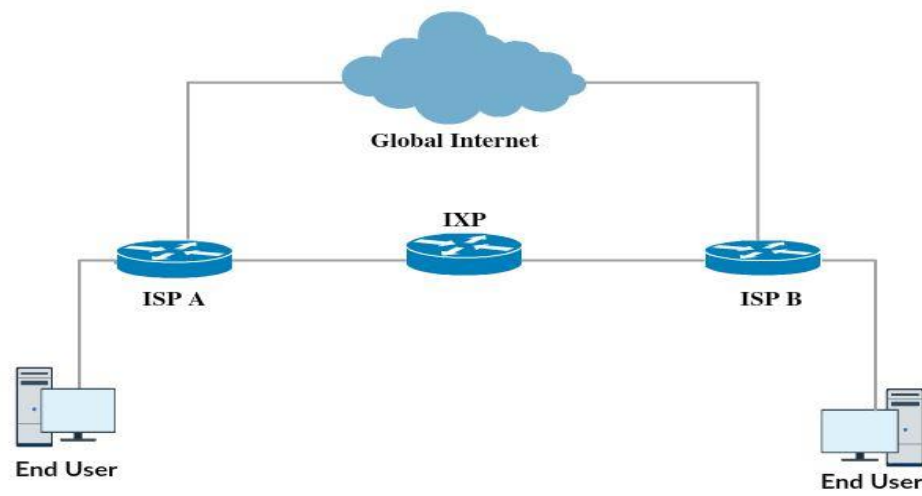

Figure 1. Basic schematic diagram of an IXP 
As IXP ensured us better local traffic, our goal is to use local bandwidth to get over with global bandwidth syndication and ensure a thorough distribution with maximized speed of global traffic for a decent amount of money. The details of the protocol are explained in the 'System Design and Implementation' section i.e. Section 5.

\section{BACKGROUND}

Starting in the early 1990s, Bangladesh had dialup access to e-mail using the Bulletin Board Systems (BBSs) of a few local providers, but the number of users was not more than 500. Users were charged by the kilobyte and email was transferred from the BBS service providers to the rest of the world by international dialup using UUCP (Unix-to-Unix Copy).

Bangladesh is passing through some massive technological changes. From nineties' VSAT communication system to today's Internet accessibility through the submarine cables, our country's communication pattern faced many challenges and successfully overcame those obstacles by adopting $3 \mathrm{G}$, Wi-Max and LTE technologies. After introducing the submarine cable system, the number of local ISPs grew up drastically. Before the introduction of IXP, local traffic had to travel to other countries to reach its final destination. Therefore, prior infrastructure was not able to provide a cost-effective service. The internet penetration of Bangladesh is one of the lowest in the world, only greater than North Korea, Myanmar and Sierra Leon which are exceptional cases [12]. In one of the press releases, the CEO of Grameenphone enlighten us with impressive information. He forecasts that by the year 2020, the total number of internet user in Bangladesh will be nearly 20 million. An estimate of internet users and their growth is shown in Table $1[3,4,19]$. We see in the year 2010 , only $3.68 \%$ of the total population was using the Internet. By the year 2018, the number of Internet users increases to 54.9\% which is around 15 times of the year 2010. The BTCL, WiMAX operators, mobile operators and numerous ISPs are trying to reach every corner of the country to expand their services to make the internet a daily useful companion. According to BTRC (Bangladesh Telecommunication Regulatory Commission), the total number of Internet Subscribers has reached 91.348 Million at the end of December 2018 [19]. Table 2 shows statistics of Internet subscribers in Bangladesh.

As Internet Exchange Point has a profound impact over local traffic, we propose a method where local traffic can be used for elevated global traffic by creating a third wheel [2]. The third wheel uses Virtual Private Network (VPN) or proxy server. If a user has higher local bandwidth than global bandwidth, our method can establish an elevated global traffic route by counting little extra money to VPN providers.

Table 1. An Estimate of Internet Users and Their Growth

\begin{tabular}{cccc}
\hline Year & Number of users & Population & \% Penetration \\
\hline 2000 & 93,261 & $131,280,739$ & $0.07 \%$ \\
2005 & 345,372 & $142,929,979$ & $0.24 \%$ \\
2010 & $5,609,821$ & $151,616,777$ & $3.68 \%$ \\
2015 & 54,120000 & $161,200,886$ & $33.58 \%$ \\
2016 & 66,623000 & $162,951,560$ & $40.89 \%$ \\
2017 & 80,483000 & $164,669,751$ & $48.88 \%$ \\
2018 & 91,348000 & $166,368,149$ & $54.9 \%$ \\
\hline
\end{tabular}

Table 2. Internet Subscriber in Bangladesh

\begin{tabular}{cc}
\hline Operator & Subscriber (Million) \\
\hline Mobile Internet & 85.552 \\
WiMAX & 0.061 \\
Broadband & 5.735 \\
Total & 91.348 \\
\hline
\end{tabular}

For example, one internet provider is serving their customer with 1 Mbps Global bandwidth along with $20 \mathrm{Mbps}$ of local bandwidth for 500 BDT (Bangladesh Taka). On the other hand, another provider is providing his customer with $5 \mathrm{Mbps}$ of global bandwidth and $30 \mathrm{Mbps}$ of local bandwidth for the same amount of money. In this case, we observe both ISPs are providing sufficient bandwidth for local traffic, but their global bandwidth is not satisfactory yet. Again, there is a huge difference in global bandwidth between two ISPs. Now the first customer who is currently getting $1 \mathrm{Mbps}$ global bandwidth from his ISP can get up to $5 \mathrm{Mbps}$ global bandwidth from the second ISP using their VPN server.

\section{RELATED WORKS AND MOTIVATION}

Bangladesh Telecommunication Regulatory Commission (BTRC) has been working deliberately for making internet handy to everyone. BTRC took a quintessential step to make a revolutionary change in existing infrastructure since the day Bangladesh was connected to the international submarine cable 'SeaMeWe-4' in 2005. With existing internet bandwidth likely to be exhausted next year, the government is in talks with a consortium for a third undersea cable connection. The demand for internet bandwidth almost 
doubled in the last one year, to 1,000 gigabits per second (Gbps) from 550 Gbps of late 2017. And experts fear the country could run out of bandwidth even sooner. Industry insiders said the introduction of $4 \mathrm{G}$ mobile services in Bangladesh helped push the bandwidth demand into 1,000 gigabits per second (Gbps) from 550 Gbps of late 2017 [18]. Demand from users of wired broadband internet had been increasing as well. As carriers are now prepping for 5G in the developed world [17], it will not be too long before the fifthgeneration mobile internet service is made available in Bangladesh.

Bangladeshi ISPs were connected to its own IXP known as Bangladesh Internet Exchange (BDIX), thereby Bangladeshi users enjoying tremendous internet speed for local traffic. Without IXP local traffic has to travel to other countries to reach its final destination. So IXP has been a revolutionary technology in the world of communication as it changed the whole communication pattern of the Internet. Several notable researches and projects have been conducted for the deployment of IXP which are outlined below.

"Internet Exchange Point: A Context of Bangladesh" is one of the finest research papers based on IXP. In this paper, the authors described the role of IXP and also explained how the whole infrastructure of a county could be changed through the setup of IXP [1]. The authors also enlightened us how a cost-effective model could be achieved by the deployment of Internet Exchange Point. In 2004, Russell Southwood published an outstanding article upon the request of the International Telecommunication Union (ITU) and the International Development Research Centre (IDRC) that addresses two issues of direct relevance to most developing countries [5]. One of the factors inhibiting the growth of Internet usage in these countries is the high cost of bandwidth and the usage of this international bandwidth to exchange data at a local and national level. The other being: the need to setup an IXP to accelerate the growth of local traffic and save incurring international bandwidth cost.

The author of [6], [29] focuses on the problems and opportunities for IXP deployment in Africa. The paper includes two distinct case studies for Kenya and Bangladesh focusing on their local Internet traffic, cost of bandwidth and IXP deployment issues. Another paper titled "Economics of Internet Exchange Points" by Mouhamet Diop published in 2009 gives an in-depth analysis of Internet transit, internet peering ecosystem and the economic impact of an IXP in a country. The paper also discusses various issues concerning the creation and operation of an Internet Exchange and tries to come up with an appropriate business model for it [7].

Although IXPs have been shown to contribute a significant role in the evolution of the modern Internet topology, there have been only a few works in determining their effect on packet routing across the Internet. Ahmad et al. [27] put forward a way in this context investigating how IXPs affect routes to the most popular websites across the globe. The main outcome of the study is finding the bandwidth availability of links along a route traversing an IXP and the corresponding effect on end-to-end latencies. Lv et al. in [28] statistically show that the transmission speed of peer-to-peer networks in IXP is constantly increasing which is very promising in future generation communications.

\section{SYSTEM DESIGN AND IMPLEMENTATION}

In this section, at first, we delineate a general IXP system and its requirements. Then we describe and implement our proposed method of enhancing global bandwidth which is the core objective of this article.

\subsection{Apparatus and Mechanism of Internet Exchange Point}

Internet Exchange Point acts as a switch that routes traffic among its associated ISPs. It saves the bandwidth cost and time of the large intermediate portion of internet flow from source to destination. It is mandatory that all intended ISPs must be connected through a common IXP. In Figure 2 Tier 1 providers are the backbone of international traffic. Tier 2 is a thorough distributor of international traffic to Tier 3 by peering agreements. Tier 3 buys internet bandwidth from Tier 2 and provides internet connectivity to corporate and residences. Throughout the whole journey of traffic, IXP reduces the usage of global bandwidth by filtering out local traffic which previously uses the global route. Thus, the usage of international bandwidth reduces dramatically. If we investigate the top 20 websites searched by Bangladeshi users, we can see our Bangladeshi websites such as Prothom Alo, Kalerkantha, Jugantor, Bdjobs etc. are ruling in the top 20. Table 3 shows the top 20 websites which are surfed most of the time by Bangladeshi users [7]. The traffic of local websites need not travel through Tier 1 as this traffic will consume only local bandwidth using IXP. Thus, the Internet system using IXP could provide a faster connection in our country which could be implemented by any other country in the world.

For the establishment of an IXP, we require some elements such as root server, route server, NTP server, looking glass etc. [9]. These are illustrated in Figure. 3. 


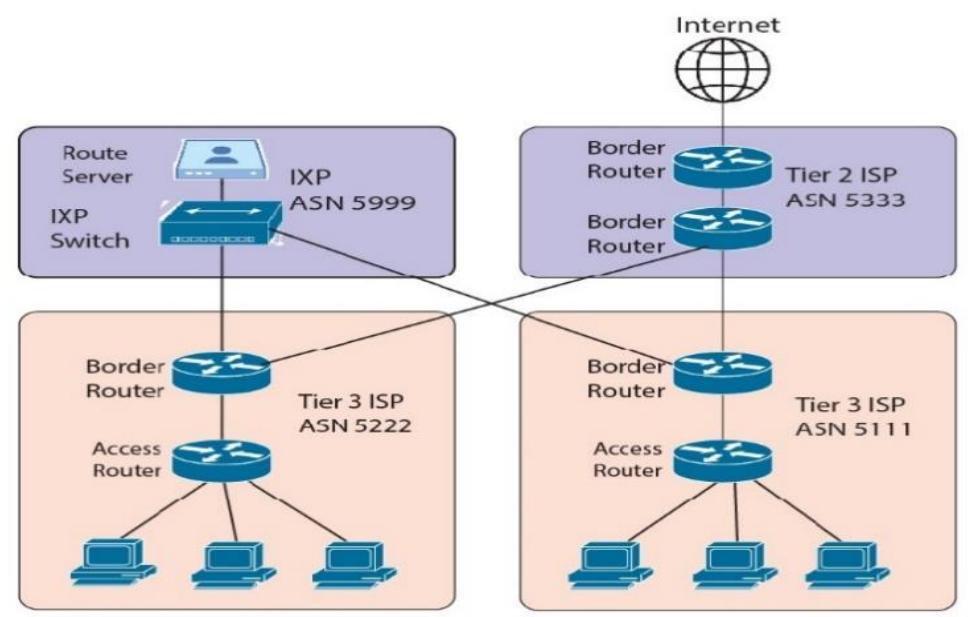

Figure 2. A typical network with an IXP in the middle

Table 3. Top 20 Website Browsers by Bangladeshi Users

\begin{tabular}{|c|c|c|c|c|c|c|}
\hline 1. & google.com & 6. & yahoo.com & 11. bd24live.com & 16. & banglanews24.com \\
\hline 2. & youtube.com & 7. & kalerkantho.com & teletalk.com.bd & 17. & 24livenewspaper.com \\
\hline 3. & facebook.com & 8. & google.com.bd & bdjobs.com & 18. & crickbuzz.com \\
\hline 4. & prothomalo.com & 9. & wikipedia.org & blogspot.com & 19. & bdnews24.com \\
\hline 5. & jugantor.com & 10. & bd-pratidin.com & jagonews24.com & 20. & daraz.com.bd \\
\hline
\end{tabular}

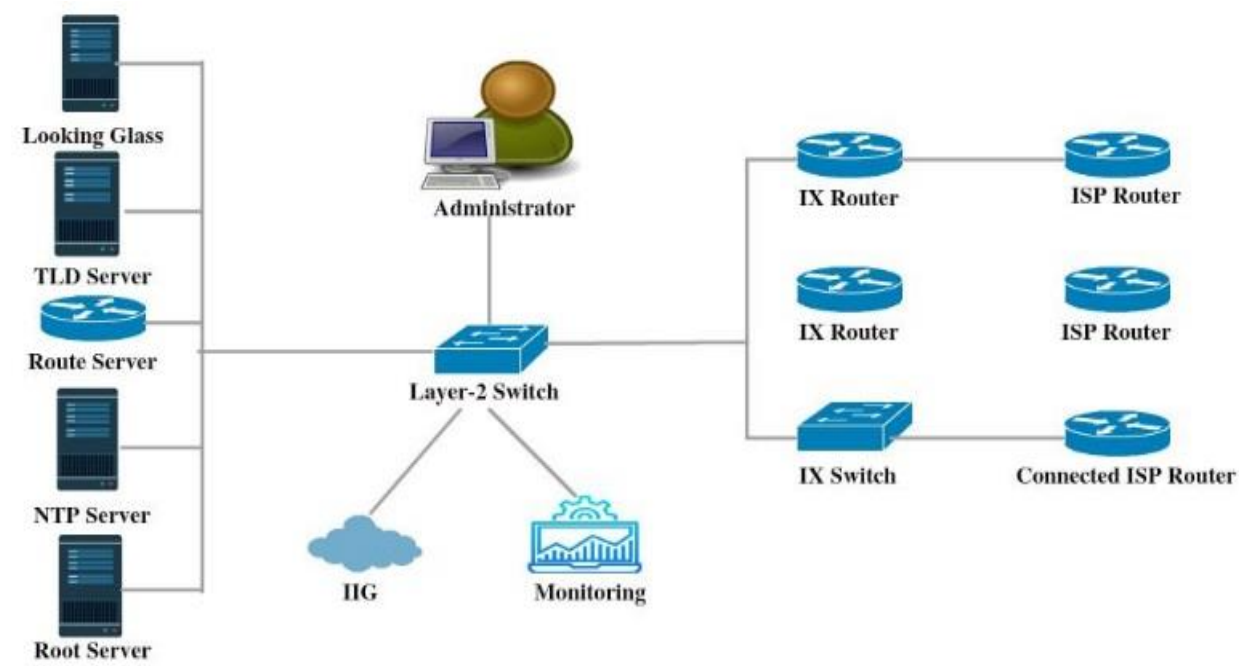

Figure 3. Schematic diagram of an IXP

\subsection{Implementation Requirement}

The first and foremost requirement is both ISPs must be connected to the common IXP. For the third wheel that helps us to accelerate the global bandwidth, we need a VPN or proxy server. For our project, we rent a Linux VPS (Virtual Private Server) from the company 'Xeon BD' where we installed a VPN server. We have determined the result using our method after connecting to VPN server. Speedtest.net [11] is the website that we use to measure the acceleration of bandwidth with a Singaporean server named 'Singtel'.

\subsection{Procedures of Implementation}

Our aim is to maximize the global bandwidth leveraging VPN technology utilizing local bandwidth. A user who is getting poor global bandwidth from his current ISP can be benefitted by an IXP to boost up his global bandwidth. For that, the user must be subscribed to another ISP that is providing higher global bandwidth from his current ISP and both ISPs must be connected to each other through the common IXP. 
Thus, a user getting a higher global bandwidth provided by his new ISP sitting in the network of his old ISP's and ISP to ISP traffic through IXP utilizes local bandwidth. The user has to take the services of a VPN or proxy server from the new ISP. If new ISP does not provide these services, then the user has to setup the VPN server by himself. When a user access the internet via a VPN server, he acts as a DHCP client to the VPN server. After connecting the VPN server, traffic between the current ISP to VPN server (new ISP) will use the user's local bandwidth portion. Now user's global traffic will be routed through the VPN server. Figure 4 illustrated the scenario.

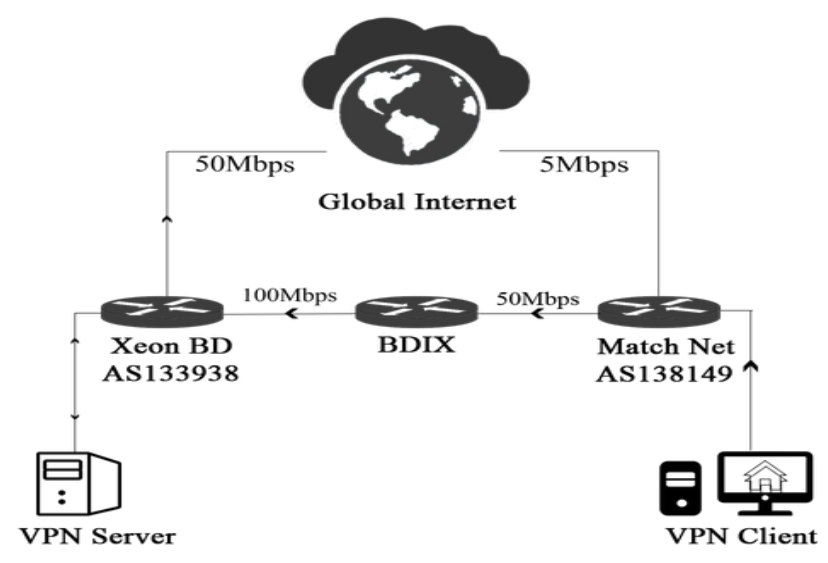

Figure 4. A typical network of the proposed method with an IXP in the middle

Initially, we have taken internet services from an ISP named 'Match Net' [20] located at Kolabagan, Dhaka that providing global bandwidth $5 \mathrm{Mbps}$ along with $50 \mathrm{Mbps}$ local bandwidth. To implement our technique, we rent a Linux VPS from another data centre named 'Xeon BD' [25] located at Mohammadpur, Dhaka. Xeon BD provides us 50 Mbps global bandwidth along with $100 \mathrm{Mbps}$ local bandwidth.

In VPS we have installed the SoftEther VPN server which supports multiple types of VPN. In the server, we enabled L2TP, IPSec, SSTP, and OpenVPN. As VPS does not contain a built-in DHCP server, we utilize virtual NAT function where we enable SecureNAT. Whenever a client connects the VPN server, the server will provide a private IP address from SecureNAT pool. In client end, we use the SoftEther VPN Client application which uses SSTP (Secure Socket Tunneling Protocol) by default to connect the VPN server.

\section{PERFORMANCE EVALUATION AND COST-BENEFIT ANALYSIS}

In this section, at first, we measure the efficiency of our developed method illustrated in the Section 5. Then we show the tremendous benefits of the project through comprehensive cost-benefit analysis and user ratings.

\subsection{Performance Evaluation and Comparison}

In this sub-section, we evaluate the performance of our proposed method and numerically determine the improvement of global bandwidth sitting at different locations of different ISP's in Dhaka City. Table 4 shows different internet package information provided by some renowned ISP of the capital city Dhaka. The data of the last column i.e., Obtained Global Internet Speed of Table 4 is measured by us using Speedtest.net website with the help of a Singaporean server 'Singtel'.

We have gathered information about five ISPs' namely Match Net [20], ICC Communication [21], Explore Online [22], Stargate Communication [23] and Mazeda Network [24]. Their global bandwidth ranges from 2-10 Mbps, local bandwidth ranges from 30-100 Mbps, package cost ranges from 500-700 BDT and our measured global speed ranges from 2.00-9.68 Mbps.

Table 5 shows the improvement of global bandwidth after using our method. To acquire higher global bandwidth, we connected our VPN server which hosted on Xeon BD from primary ISPs' user end. Again, we have tested global bandwidth by using Speedtest.net. It is noted that Xeon BD is providing a pleasing global bandwidth of 50 Mbps which is substantially larger than other ISPs'.

Examining Table 5, we can easily realize the differences in global speed using and without using an IXP and can perceive the effects of our proposed method. Now sitting at each of the primary ISP's network 
we are getting an enlarged global bandwidth which is around 4 to 8 times higher than the allocated bandwidth. Figure 5 summarizes the output of the system graphically. The blue bars represent the global bandwidth which is given by the ISPs and the orange bars represent the results after employing our method.

Table 4. Data Collected from Different ISP

\begin{tabular}{|c|c|c|c|c|}
\hline No & ISP Name & Package Specification & Package Cost & Obtained Global Internet Speed \\
\hline 01 & Match Net & $\begin{array}{l}5 \text { Mbps (Global Bandwidth) } \\
50 \text { Mbps (BDIX Bandwidth) }\end{array}$ & $500 \mathrm{BDT}$ & $3.94 \mathrm{Mbps}$ \\
\hline 02 & ICC Communication & $\begin{array}{l}4 \text { Mbps (Global Bandwidth) } \\
30 \text { Mbps (BDIX Bandwidth }\end{array}$ & $700 \mathrm{BDT}$ & $3.96 \mathrm{Mbps}$ \\
\hline 03 & Explore Online & $\begin{array}{l}10 \text { Mbps (Global Bandwidth) } \\
100 \text { Mbps (BDIX Bandwidth) }\end{array}$ & $500 \mathrm{BDT}$ & $9.68 \mathrm{Mbps}$ \\
\hline 04 & $\begin{array}{c}\text { Stargate } \\
\text { Communication }\end{array}$ & $\begin{array}{l}2 \text { Mbps (Global Bandwidth) } \\
70 \text { Mbps (BDIX Bandwidth) }\end{array}$ & $500 \mathrm{BDT}$ & 2 Mbps \\
\hline 05 & Mazeda Network & $\begin{array}{l}8 \text { Mbps (Global Bandwidth) } \\
50 \text { Mbps (BDIX Bandwidth) }\end{array}$ & $700 \mathrm{BDT}$ & $7.8 \mathrm{Mbps}$ \\
\hline
\end{tabular}

Table 5. Data Comparison Before and After Using Proposed Method

\begin{tabular}{ccccc}
\hline No & Source ISP & $\begin{array}{c}\text { Obtained Global Internet } \\
\text { Speed (Previous data) }\end{array}$ & $\begin{array}{c}\text { Obtained Global Internet Speed } \\
\text { (Applying VPN over IXP) }\end{array}$ & Improvement \\
\hline 01 & Match Net & $3.94 \mathrm{Mbps}$ & $30.55 \mathrm{Mbps}$ & 7.75 times faster \\
02 & ICC Communication & $3.96 \mathrm{Mbps}$ & $27.48 \mathrm{Mbps}$ & 6.94 times faster \\
03 & Explore Online & $9.68 \mathrm{Mbps}$ & $41.43 \mathrm{Mbps}$ & 4.27 times faster \\
04 & Stargate Communication & $2 \mathrm{Mbps}$ & $16.14 \mathrm{Mbps}$ & 8.07 times faster \\
05 & Mazeda Network & $7.8 \mathrm{Mbps}$ & $31.25 \mathrm{Mbps}$ & 4.00 times faster \\
\hline
\end{tabular}

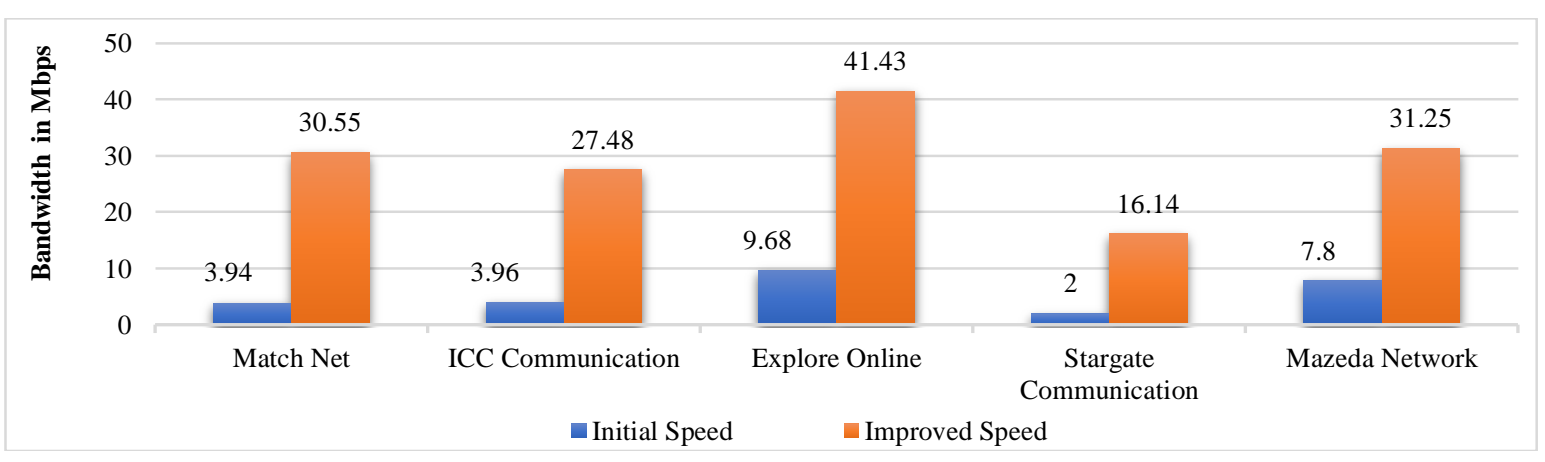

Figure 5. Enhancement of global bandwidth

\subsection{Cost-Benefit Analysis}

Analyzing cost benefit, we can show that the bandwidth price will be significantly reduced after implementing VPN technology. According to Table 4 and Table 5, we roughly estimate that after employing VPN, on an average our cost increases around 1.7 times while speed increases 6.2 times. Thus, we must acknowledge the tremendous benefit of employing IXP leveraging VPN technology.

In Table 6 we have shown the details of cost-benefit analysis. Column 3 of the table is showing the cost and obtained global speed (measured by us) of basic/primary packages of our experimenting 5 ISPs'. Column 4 is showing the cost and obtained enhanced global speed of the basic packages employing our proposed method (utilizing IXP using VPN). The cost in column 4 is the summation basic package cost of the corresponding ISP and VPN cost. We already mentioned that we have rented VPS server from 'Xeon BD' for 400 BDT. It is noted that ISPs also provide high-speed packages other than the basic/initial package. These high-speed packages have low cost (considering taka per megabyte) than basic package. Thus, to get exact cost savings (shown in column 6) we consider high speed package which is equivalent (in terms of speed) to our enhanced bandwidth rather than considering the basic package.

For example, Match Net provides a basic package having a speed of 3.94 Mbps for 500 BDT. Implementing VPN over IXP on this connection we got a global speed of $30.55 \mathrm{Mbps}$ for 900 BDT (Basic package cost of Match Net 500 BDT + VPS cost of Xeon BD 400 BDT). If we take a high-speed package having a speed of $30.55 \mathrm{Mbps}$ from Match Net, it will cost 2000 BDT. Thus, we save $(2000-900)=$ 
1100 BDT which is $122 \%$ of 900 BDT. Similarly, we can save $355 \%, 344 \%, 344 \%$ and $155 \%$ on ICC Communication, Explore Online, Stargate Communication and Mazeda Network packages respectively.

Table 6. Data Derived from Cost-Benefit Analysis

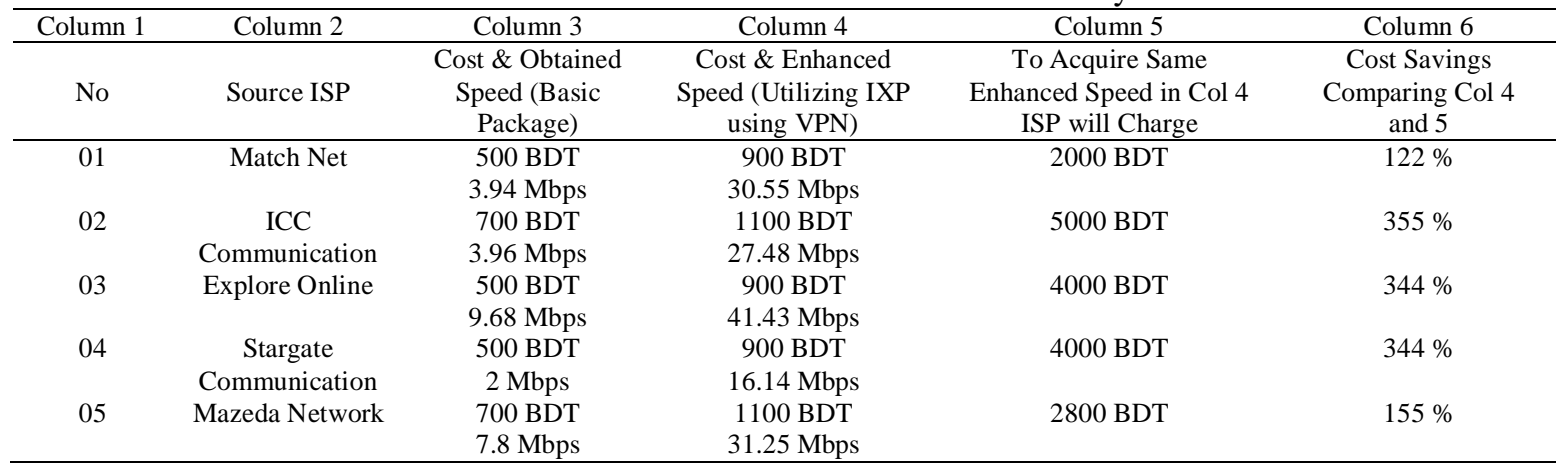

\subsection{User Ratings}

To perceive the users' acceptability or satisfaction of our proposed VPN method, it is urged to conduct a survey on the selected population of Dhaka City. Hence, we conduct a survey to get feedback of the Internet users. This survey reflects their experiences on browsing the network using different packages provided by different ISPs with and without using our method.

There are three 3 types of services/options/packages available to the users. As mentioned in Table 6 the packages are:

a) Basic Package (mentioned in Column 3)

b) Basic Package with VPN (mentioned in Column 4)

c) High-speed Package (mentioned in Column 5)

We collected feedbacks from 113 samples (Internet users) from the Internet users living in different areas in the City of Dhaka. Among 113 samples, 20 using Match Net, 23 using ICC Communication, 27 using Explore Online, 18 using Stargate Communication and 25 using Mazeda Network services. The users could rate each of the services/options/packages on a scale of 0 to 5 (worst to best). Then we calculate the average of the ratings provided by the users. The selected dwellers of the Dhaka City have rated different packages of different ISPs which are summarized in the bar chart shown in Figure 6.

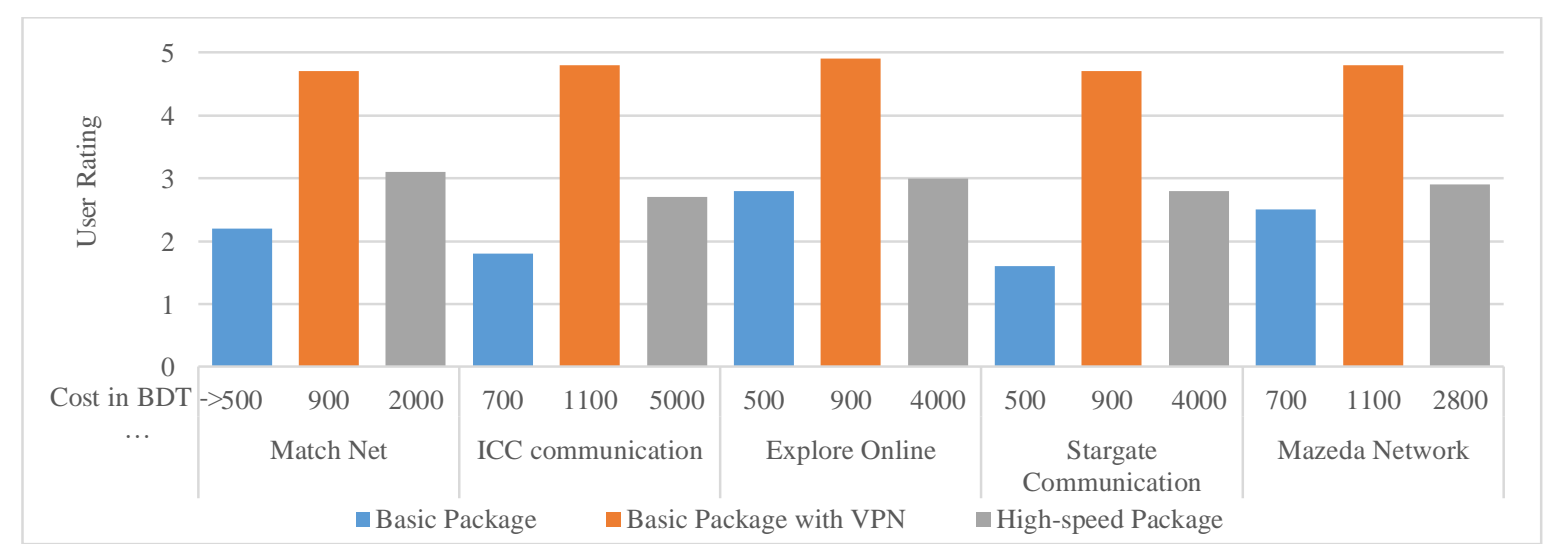

Figure 6. User ratings for 3 types of packages for different ISPs

Examining the chart, we see the users first choice is 'Basic Package with VPN' which got the highest rating i.e., 4.7 for Match Net, 4.8 for ICC Communication, 4.9 for Explore Online, 4.7 for Stargate Communication and 4.8 for Mazeda Network. Thus, our method is the most attractive to the users due to the tremendous speed at low cost. The second choice is 'High-speed Package' which has an average rating between 2.7 to 3.1 for different ISPs. Among the three packages/options 'Basic Package' has the lowest rating ranging from 1.6 to 2.8 due to its high cost and low speed. 


\section{BYPASSING FIREWALL RESTRICTION}

Our primary objective of this project is to provide high-speed Internet to the browsers which is already implemented in Section 5. Our second objective is to facilitate the user's unrestricted access to the Internet ocean along with robust bandwidth. Thus, in this section, we incorporate the idea of bypassing georestriction to the proposed bandwidth enhancement mechanism.

Primary VPN usually routes all internet traffic through an encrypted tunnel to protect data from hackers or any third parties. When we have different bandwidth allocation for local and global routes i.e. ISP might allocate us $100 \mathrm{Mbps}$ local traffic and $10 \mathrm{Mbps}$ global traffic, we are able to bypass that global bandwidth cap following our previous method explained in Section 5. In this connection, we are also going to implement a firewall bypassing technique using a secondary VPN along with our existing primary VPN. This firewall bypassing method is a smart solution when the authority in a region (Government, ISP etc.) blocked some websites or IPs. It is noted, we can bypass firewall restriction independently (i.e. not merging with our proposed global bandwidth enhancement technique) just connecting a VPN that is hosted outside our region. But that method is not promising as global bandwidth is inherently low. To access restricted websites along with achieving higher global bandwidth, we establish a secondary VPN (Digital Ocean) connection through our primary VPN (Xeon BD). Thus, primary VPN allows us enhanced global bandwidth while secondary VPN allows us bypassing firewall restriction. A typical network for firewall bypassing with our proposed bandwidth enhancement model is shown in Figure 7.

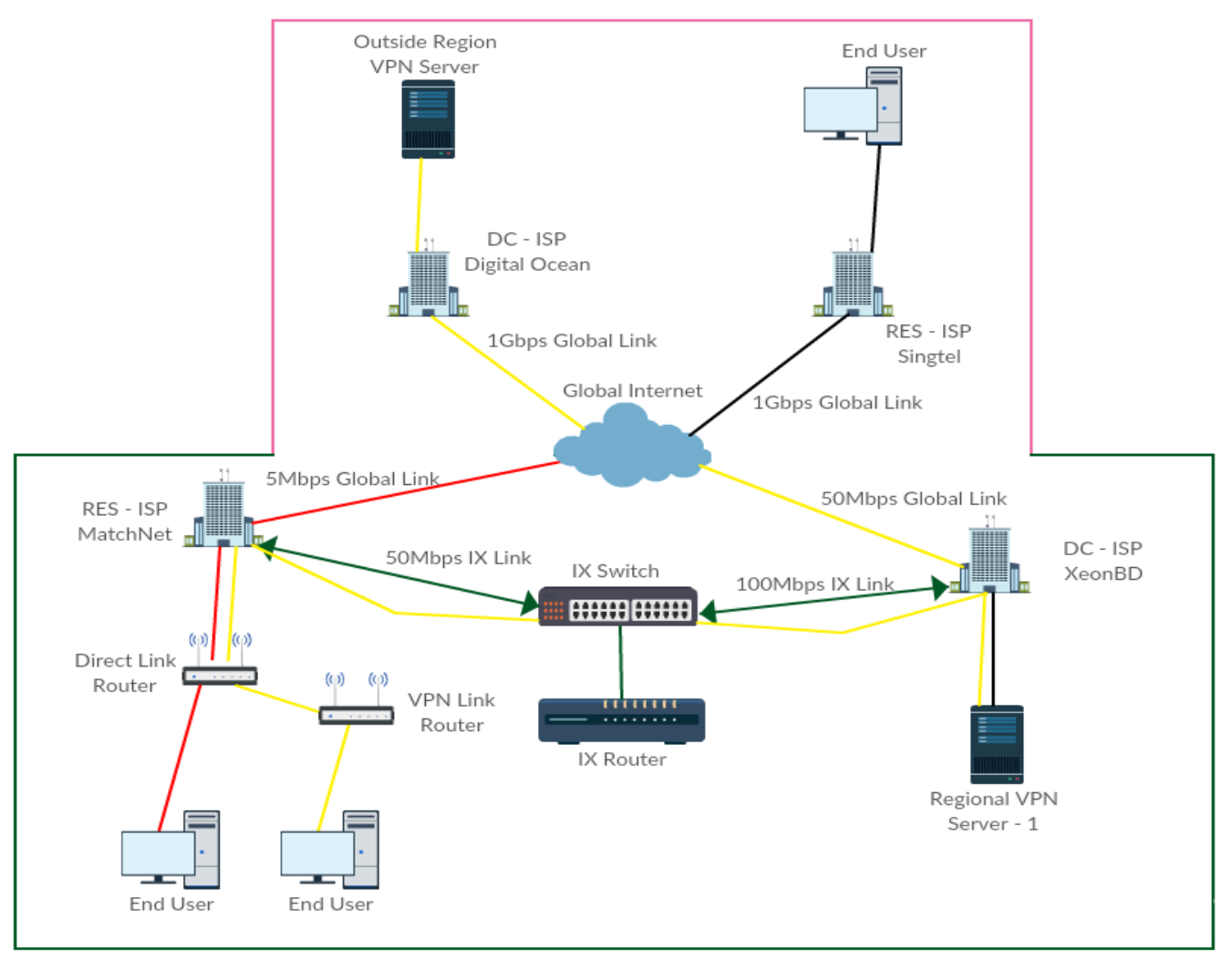

Figure 7. A typical network for firewall bypassing with our proposed bandwidth enhancement model

Previously we installed SoftEther VPN server on our datacenter-based secondary ISP 'Xeon BD'. Since 'Xeon BD' cannot bypass firewall restriction, we rented a VPS subscription from a datacenter-based ISP named 'Digital Ocean' located in Singapore [26] which is not firewall restricted. In Digital Ocean, we setup a VPN server as we have done before in Xeon BD. To create a chain VPN and route to one single connection, we followed the easiest possible technique. In our home network, we use a Raspberry Pi 3 Model B where we setup it as a client of our primary VPN server (Xeon BD). Then we configure Raspberry Pi to act a Wi-Fi hotspot of primary VPN connection. From another device, we connect to that Wi-Fi hotspot and initiate VPN connection with the secondary VPN server. Thus, we can enjoy tremendous global bandwidth with unrestricted access to the Internet. 


\section{CONSTRAINTS AND RECOMMENDATIONS}

There are some constraints and limitations to implementing the project using our intended method. Our method will work if and only if the ISPs belong to a common IXP. For example, if one ISP only belongs to BDIX and another one only belongs to "NOVOCOM NIX" then the method will be void. Another thing is that local bandwidth of all ISPs must be good enough otherwise a bottleneck would occur. Lastly, users who want to experience an elevated global bandwidth must have to buy a VPN server from the ISP that providing a robust global bandwidth.

Another important thing is that while we implement firewall bypassing as well as bandwidth enhancement, we are employing multi-tunneling (i.e. chain VPN) procedure to route the traffic. In the multitunneling procedure, internet speed drops significantly due to double encryption. By the way, this double tunneling ensures the highest level of security.

\section{CONCLUSION}

The Internet has been a tremendous gateway for acquiring knowledge as well as a constant companion for entertainment. Every user desires a high-speed internet connection at a lower price as well as access to all sites around the world. However, most of the citizen especially from developing countries are not getting these benefits adequately. To resolve the problems, we have undertaken this project concerning the residents of Dhaka City. Thus, our main goal is to implement a method that can overcome bandwidth syndication, capping of bandwidth and provide every user a pleasant experience of using Internet with a maximized global bandwidth without paying large amount money. Our secondary goal is to provide the users access to the politically or ill-fully motivated blocked sites.

In this article, we effectively illustrate a project that boosts global bandwidth using IXP leveraging VPN technology. We also integrate a firewall bypassing technique along with the enhancement of bandwidth. Therefore, our method can be used to resolve the syndication problem, deficiencies of internet speed and give comfort to the users for the next generation communications.

\section{REFERENCES}

[1] Md. Shakilur Rahman, Muhtasim Iqbal and Nova Ahmed, "Internet Exchange Points: A Context of Bangladesh", 14th International Conference on Computer and Information Technology (ICCIT 2011), December 2011.

[2] M. Z. Ahmad and R. Guha, "Studying the effect of internet exchange points on internet link delays," Proceedings of the Communications and Networking Symposium (CNS), SCS SpringSim 2010, Orlando, FL, April 2010.

[3] Internetlivestats.com, "Bangladesh Internet Users", March 2018. [Online]. Available: http://www.internetlivestats.com/internet-users/bangladesh.html. [Accessed: 20- Nov- 2018].

[4] Worldmeters.info, "Bangladesh Population", January 15, 2019. [Online]. Available: http://www.worldometers.info/world-population/bangladesh-population. [Accessed: 22- Mar- 2019].

[5] Russell Southwood, "Via Africa Creating local and regional IXPs to save money and bandwidth", Global Symposium for Regulators by International Telecommunication Union, 2004.

[6] Andrew McLaughlin, "Internet Exchange Points Their Importance to Development of the Internet and Strategies for their Deployment-The African Example", Global Internet Policy Initiative, May 2004.

[7] Mouhamet Diop, "Economics of Internet Exchange Points", Afrinic 10 Cairo, May 2009.

[8] Alexa.com, "Top sites in Bangladesh", January 8, 2018. [Online]. Available: https://www.alexa.com/topsites/countries/BD [Accessed: 15- Aug- 2018].

[9] Btrc.gov.bd, "BTRC NIX Licensing Guidelines (Draft)", March 16, 2018. [Online]. Available: www.btrc.gov.bd/sites/default/files/nix_ guide0.pdf. [Accessed: 20- Oct- 2018].

[10] Bdix.net, "Bangladesh Internet Exchange (BDIX)", April 05, 2018. [Online]. Available: http://www.bdix.net. [Accessed: 30- Oct- 2018].

[11] Speedtest.net, "Speedtest by Ookla-The Global Broadband Speed Test", January 06, 2018. [Online]. Available: http://www.speedtest.net. [Accessed: 29- Dec- 2018].

[12] Wikipedia.com, "Telecommunications in Bangladesh", 2018. [Online]. Available: https://en.wikipedia.org/wiki/Telecommunications_in_Bangladesh. html. [Accessed: 18- Dec- 2018].

[13] K. Xu, Z. Duan, Z. L. Zhang and J. Chandrashekar, "On properties of internet exchange points and their impact on as topology and relationship," Networking, vol. 3042, 2002.

[14] Y. He, G. Siganos, M. Faloutsos and S. Krishnamurthy, "Lord of the links: a framework for discovering missing links in the internet topology," IEEE/ACM Trans. Networking, vol. 17, no. 2, pp. 391-404, 2009.

[15] Sciencedirect.com, "Exchange Point", January 05, 2019. [Online]. Available: http://www.sciencedirect.com/topics/computer-science/excha nge-point. [Accessed: 30- Feb- 2019].

[16] Cloudflare.com, "Content Delivery Network", Nov 06, 2018. [Online]. Available: http://www.cloudflare.com/ learning/cdn/what-is-a-cdn. [Accessed: 02- Jan- 2019].

[17] Gazi Zahirul Islam and Mohammod Abul Kashem, "An OFDMA-based new MAC mechanism for IEEE 802.11ax," 5th International Conference on Networking, Systems and Security (NSysS), pp. 1-7, December 2018. 
[18] Muhammad Zahidul Islam, "Push for the third submarine cable," The Daily Star, para. 2, February $20,2019$. [Online]. Available: https://www.thedailystar.net/frontpage/news/push-the-third-submarine-cable-1704691. [Accessed March. 18, 2019].

[19] Btrc.gov.bd, "Internet subscribers in Bangladesh (2010-2018)", January 13, 2019. [Online]. Available: http://www.btrc.gov.bd/telco/internet. [Accessed: 13- Mar- 2019].

[20] Matchnetbd.com, "Matchnet Internet Service Provider", February 01, 2019. [Online]. Available: https://matchnetbd.com. [Accessed: 21- Mar- 2019].

[21] Icc.com.bd, "ICC Communication LTD.", February 03, 2019. [Online]. Available: http://icc.com.bd/. [Accessed: 23- Mar- 2019].

[22] Exploreonlinebd.com, "Explore Online", February 07, 2019. [Online]. Available: http://exploreonlinebd.net/. [Accessed: 25- Mar- 2019].

[23] Stargatebd.com, “Stargate Communication LTD.”, February 08, 2019. [Online]. Available: https://stargatebd.com/. [Accessed: 28- Mar- 2019].

[24] Mazedanetworks.net, "Mazeda Network LTD.", February 10, 2019. [Online]. Available: http://www.mazedanetworks.net/. [Accessed: 28- Mar- 2019].

[25] Xeonbd.com, "XeonBD”, February 11, 2019. [Online]. Available: https://www.xeonbd.com. [Accessed: 27- Mar2019].

[26] Digitalocean.com, "DigitalOcean-The developer cloud", February 18, 2019. [Online]. Available: https://www.digitalocean.com. [Accessed: 17- Mar- 2019].

[27] Mohammad Zubair Ahmad and Ratan Guha, "A measurement study determining the effect of Internet eXchange Points on popular webservers," 3rd IEEE International Workshop on Wireless and Internet Services, Denver, CO, USA, October 2010.

[28] Ting Lv, Donghong Qin, Lina Ge, "Research and Analysis of Statistical Characteristics of Internet Exchange Points", International Symposium on Computing and Networking Workshops, Japan, November 2018.

[29] Gazi Zahirul Islam, "E-Governance Implementation in Metropolitan and Rural Areas of Bangladesh: An Illustrative Prototype," International Technology Management Review, Vol. 2, No. 1, pp. 33-49, October 2009.

[30] Md. Abbas Ali khan et.at., "A detailed investigation of the impact of online transportation on Bangladesh economy," Indonesian Journal of Electrical Engineering and Computer Science Vol. 16, No. 1, October 2019, pp. 420-428.

[31] Teddy Surya Gunawan et. al., "Prototype Design of Smart Home System using Internet of Things," Indonesian Journal of Electrical Engineering and Computer Science (IJEECS), Vol. 7, No. 1, July 2017, pp. 107-115.

\section{BIOGRAPHIES OF AUTHORS}
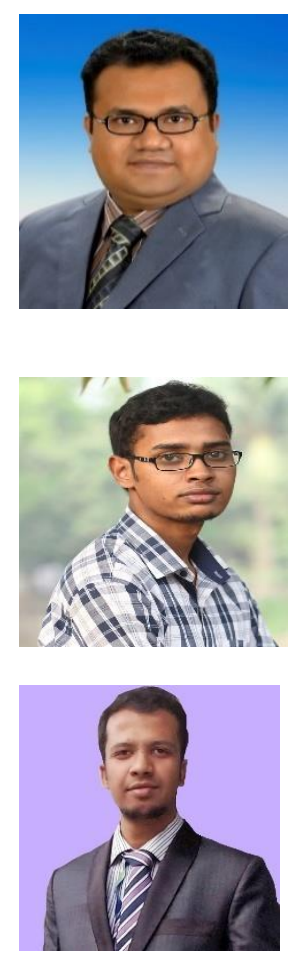

Gazi Zahirul Islam is currently pursuing his $\mathrm{PhD}$ at Bangladesh University of Professionals. He completed M.Sc. in wireless communications systems engineering from the University of Greenwich, UK and B.Sc. in computer science and engineering from Chittagong University of Engineering and Technology (CUET), Bangladesh. He has been teaching as an Assistant Professor at Daffodil International University, Bangladesh for about five years. Previously he taught at City University, Southern University Bangladesh and Primeasia University. He is an author of more than 10 research articles on wireless communications, computer networking and e-governance.

Aman Ullah Juman is currently working in the field of Network and System Administration. He completed B.Sc. in Computer Science and Engineering from the Daffodil International University in 2019. He has vast experience in configuring Amazon Web Services, cloud service, Windows Server etc. He has been working as a freelancer for system administration and possess eight years of experience in server-side work. His interested working areas are Network \& System Administration, Security Specialization and Cloud Computing Geek etc.

Al Nahian Bin Emran is currently working in Smart Living Technologies LTD in Dhaka, Bangladesh as IT Manager. He completed B.Sc. in Computer Science and Engineering from the Daffodil International University in 2019. He has several publications in the field of Information and Communication Technology. His interested research fields are Network Security, Blockchain, Wireless and Mobile Communication, Fog computing, Water Marking etc. 


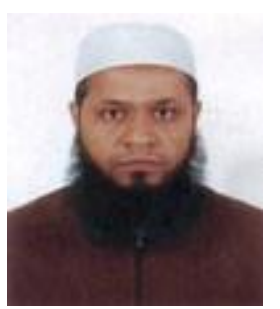

Md. Abbas Ali Khan is currently pursuing his $\mathrm{PhD}$ at Jahangirnagar University, Bangladesh and received his M.Sc. in Computer Networks from School of Technology \& Health, KTH Royal Institute of Technology, Stockholm, Sweden and B.Sc. in Computer Science from Daffodil International University, Dhaka, Bangladesh. Mr. Khan has been teaching as a Lecturer at Daffodil International University since 2017. He has published research papers both in National and International refereed journals. $\mathrm{He}$ is an Associate Member of Bangladesh Computer Society.

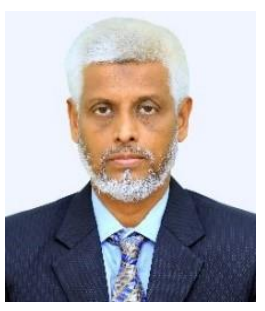

Md. Fokhray Hossain is a Professor in the Department of Computer Science and Engineering at Daffodil International University, Dhaka, Bangladesh. He has more than 27 years of teaching and research experience in Information System, Computer Architecture, Enterprise Networking, Telecommunication, Decision Support System, Management Information System and so on. He had completed his PhD from University of Glamorgan, UK in 1998 through ODASSS scholarship. He also completed M.Sc. and B.Sc. (Hon) in Physics in 1990 and 1989 respectively from Jahangirnagar University, Bangladesh. He was appointed as a research fellow in Boss Center at Dhaka University from 1991 to 1993 . He has more than 35 research publications in international journals and conferences.

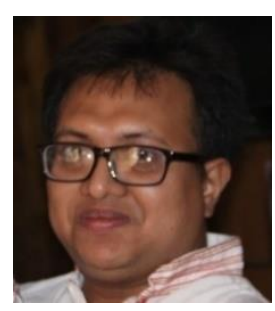

Md. Tarek Habib is pursuing his Ph.D. degree in the Department of Computer Science and Engineering at Jahangirnagar University. He obtained his M.S. degree in Computer Science and Engineering (Major in Intelligent Systems Engineering) and B.Sc. degree in Computer Science from North South University in 2009 and BRAC University in 2006, respectively. He is currently an Assistant Professor in the Department of Computer Science and Engineering at Daffodil International University. His research interest is in Artificial Intelligence, Computer Networks and E-Commerce. Ha has published a good number of articles in international journals and conference proceedings. 